\title{
Tidal Marsh Plant Community Development within Four Restored Lowland Estuaries in the Western Peninsulas of Washington State, USA
}

\author{
Shannon M. Call, Jenise M. Bauman* \\ Huxley College of the Environment, Western Washington University, Bellingham, Washington, USA

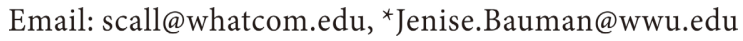

How to cite this paper: Call, S.M. and Bauman, J.M. (2021) Tidal Marsh Plant Community Development within Four Restored Lowland Estuaries in the Western Peninsulas of Washington State, USA. American Journal of Plant Sciences, 12, 1543-1558. https://doi.org/10.4236/ajps.2021.1210109

Received: August 28, 2021

Accepted: October 19, 2021

Published: October 22, 2021

Copyright ( 2021 by author(s) and Scientific Research Publishing Inc. This work is licensed under the Creative Commons Attribution International License (CC BY 4.0).

http://creativecommons.org/licenses/by/4.0/ (c) (i) Open Access

\begin{abstract}
Vegetation and soil within estuarine ecosystems play an integral role in ecological processes within pocket estuaries. However, physical barriers, caused by culverts diminish hydrological inputs, sediment exchange, and habitat connectivity. The restoration of estuaries by bridge replacement reconnects the aquatic corridor, however, the recovery of plant communities and soil substrate is not well understood. This observational study monitored four estuary restoration sites of variable ages (0 - 12 years) in Western Washington, USA. Plant community composition, soil organic carbon, organic matter, and soil nutrients were assessed. Percent soil carbon was different among the prerestoration and youngest (3-year) post-restoration site $(P=0.03)$, suggesting an initial decrease in carbon and organic matter during restoration. Both $\mathrm{N}$ and $\mathrm{P}$ were deficient at the newest, lower restoration site, presumably linked to the lack of organic matter required for adequate cation exchange capacity and nutrient/plant exchange $(P<0.05)$. Plant species diversity was higher at the intermediate (9-year) and oldest post-restoration sites (12-year; $P=0.02$ ). Vegetation composition was primarily native species with few invasive plants present. The results of this study illustrate that tidal marsh plant communities are influenced by the development of salinity and vertical gradients with older sites having an increase in species diversity. Future surveys are ongoing to better understand how these sites recover organic matter and tidal marsh communities to form adequate estuarine habitat over time.
\end{abstract}

\section{Keywords}

Estuary Restoration, Habitat Recovery, Culvert Removal, Bridge Replacement 


\section{Introduction}

Estuarine ecosystems are the link between freshwater and marine environments and are considered one of the most productive systems on the planet [1]. Hydrogeomorphology, vegetation, and soil are all functionally interrelated and facilitate energy production, biogeochemical cycling, and gene flow within habitats [2] [3] [4]. Within estuarine wetlands, sediment and vegetation play integral roles in denitrification, carbon sequestration, and physical stability through sediment stabilization and organic matter production [5] [6] [7]. These estuarine processes support valuable ecosystem services including flood mitigation, storm abatement, water quality and quantity improvements, and wetland and aquifer recharge [8]. The ecological complexity of estuary vegetation and sediment accretion also forms intricate food webs, thereby creating essential habitat for many aquatic and terrestrial wildlife species. This is of particular importance for the conservation and restoration of habitat required to support many marine, freshwater, and terrestrial wildlife species.

Despite their importance, $74 \%$ of estuary habitat in the Pacific Northwest has been lost to maritime industries, private landownership, and commercial development [9]. In Washington and Oregon alone, up to 5000 streams with over 196,000 kilometers of roadways are affected by culverts that have severely altered hydrological regimes. Loss of connectivity through the implementation of physical barriers deeply channelizes streams, diminishes flood plains, and interrupts gene flow for many species [10]. Declining shellfish, salmon (Oncorhynchus spp.), and resident Orca (Orcinus orca) populations have catalyzed an emphasis to restore estuary habitat by culvert removal and bridge replacement. It is hypothesized that the restoration of estuaries will promote energy movement and the biophysical processes required for sediment exchange, the creation of flood plains, tidal marsh succession, and improved stream morphology [11] [12] [13]. Therefore, successful estuary restoration will improve migration routes and tidal marsh habitat required to restore nursery grounds and food webs for many iconic wildlife species [14].

Due to the wide variety of restoration outcomes, effective monitoring becomes an essential component to assessing ecosystem recovery [15]. Because native tidal marsh habitat is an aspect of estuary restoration, the early detection and eradication of invasive plant species are required to encourage resilient plant communities that resemble native reference sites. Secondary disturbances during culvert restoration results in newly disturbed estuarine sediments that may lack the abiotic and biotic complexity required for native plant establishment [16]. These disturbed substrates tend to promote fast-growing exotic plant species that could impact processes such as marsh plant succession, soil development, and water relations [17] [18]. Some sites may become so invaded and degraded that they are transformed into novel systems incapable of obtaining the level of tidal marsh functioning required for estuary recovery [19]. Therefore, restoration should emphasize early detection and management of exotic species before they 
get well-established in restored estuary projects [15].

The objective of this current project is to investigate the development of soil carbon, soil organic matter (SOM), plant nutrients, and plant communities within various urban estuary restoration sites in Western Washington, USA. This observational study monitored four estuary restoration sites of variable ages (0 - 12 years) in Western Washington, USA: 1) pre-restored culverted site, 2) three-yearold restored estuary, 3) nine-year-old restored estuary, and 4) 12-year-old restored estuary. In doing so, this study will contribute information regarding soil development in various stages of estuary recovery. Additionally, this study will provide early exotic plant species detection and baseline data for long-term study of restored tidal marsh plant communities within the Kitsap Peninsula of Washington State.

\section{Material and Methods}

\subsection{Study Sites}

The four study sites are located on the Kitsap Peninsula in Washington State (Figure 1). The four sites selected included: The first site is the pre-site, Harper Creek Estuary $\left(47^{\circ} 30^{\prime} 00.9^{\prime \prime} \mathrm{N} 122^{\circ} 30^{\prime} 58.4^{\prime \prime} \mathrm{W}\right)$ in Port Orchard, Washington. This pre-restoration site within this study contained two working culverts $(60 \mathrm{~cm}$ in diameter). Soil directly adjacent to this site is composed of Tacoma silt loam and Harstine gravelly ashy sandy loam [20]. The second site, Carpenter Creek Estuary $\left(47^{\circ} 47^{\prime} 42.471^{\prime \prime} \mathrm{N} 122^{\circ} 30^{\prime} 26.7114^{\prime \prime} \mathrm{W}\right)$, located in Kingston, Washington, was restored in 2013 after removal of a $3 \mathrm{~m} \times 3 \mathrm{~m}$ box culvert on South Kingston Road and installation of a $27.4 \mathrm{~m}$ bridge that spans the entire channel width of Carpenter Creek and can withstand natural tidal inundation from Appletree Cove. The majority of soil adjacent to this site is classified as an alluvial beach deposit with minor components of Poulsbo gravelly sandy loam [20]. The third site sampled was the nine-year-old site at the Beaver Creek Estuary $\left(47^{\circ} 34^{\prime} 12.1938^{\prime \prime} \mathrm{N}\right.$ $\left.122^{\circ} 33^{\prime} 7.2468^{\prime \prime} \mathrm{W}\right)$, located in Manchester, Washington, at the head of Clam Bay and was restored in 2007. Restoration of Beaver Creek with the removal of the lower culvert and installation of a $6.7 \mathrm{~m}$ bottomless arch bridge at the head of Clam Bay were completed in 2007. Soil in this region is composed of Kapowsin gravelly ashy loam [20]. The forth site was the 12-year-old site located at the Dogfish Creek Estuary ( $\left.47^{\circ} 44^{\prime} 48.3144^{\prime \prime N} 122^{\circ} 39^{\prime} 7.3368^{\prime \prime W}\right)$, located in Poulsbo, Washington at the head of Liberty Bay. This site was restored in 2004 with the removal of a $1.5 \mathrm{~m}$ culvert, which was replaced with a $26.8 \mathrm{~m}$ bridge. Soil composition adjacent to Dogfish Creek includes urban land-Alderwood complex and Kitsap silt loam [20].

\subsection{Vegetation Community Survey}

Transects were placed parallel to the water along the lower bank edge where the first perennial vegetation was located nearest the edge of water, as described in the PacFish InFish Biological Opinion Monitoring Program [21]. Three, 50-m 


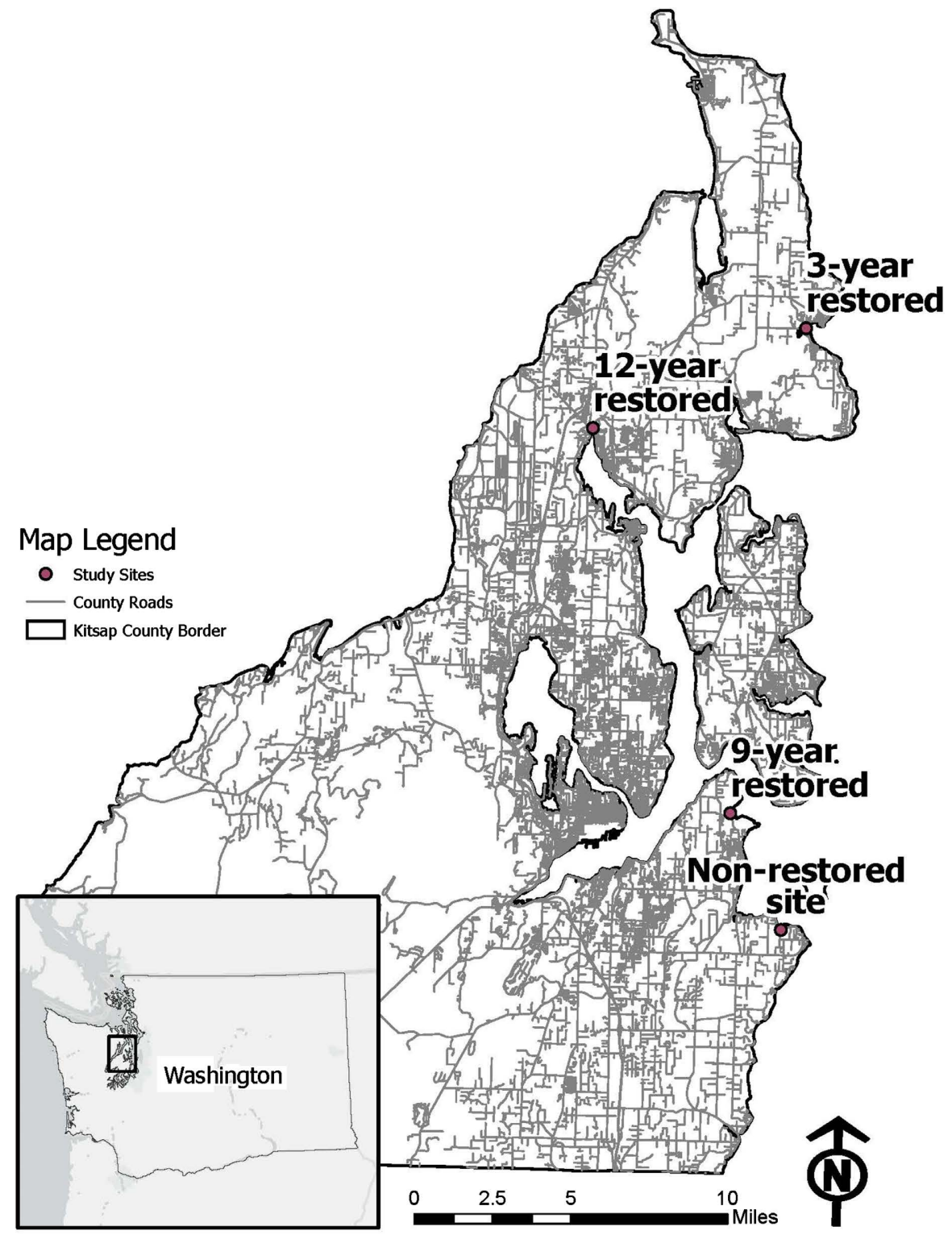

Figure 1. Map of study sites in Kitsap County, Washington State. Located on the Olympic Peninsula, sites within this study include Harper Creek (pre-restoration), Carpenter Creek (3-Yr), Beaver Creek (9-Yr), and Dogfish Creek (12-Yr). Map provided by Andrew McCay.

transects were place above the headwater of the restoration location on the freshwater influence side and three 50-m transects were placed below the tailwater on the marine influence side. The initial transect began at the bridge and subsequent transects followed the remaining estuarine vegetation, along both sides of the stream channel. Line-point intercept data was recorded along each meter of each transect wherein the species the nearest stem, leaf, or plant base intercepted was recorded using a four-letter code based on the first two letters of the genus and species. A total of 48 transects were completed in this study (4 sites $\times 2$ visits $\times 6$ transects). Along each transect, three $1 \mathrm{~m} \times 1 \mathrm{~m}$ quadrats were randomly placed (4 sites $\times 6$ transects $\times 3$ quadrats). Within each quadrat, soil 
was collected and vegetation height $(\mathrm{cm})$ from the center and each corner, along with visual percent cover of each species present was recorded. A $25 \mathrm{~cm} \times 25 \mathrm{~cm}$ sample of biomass, including all plant matter one $\mathrm{cm}$ above the soil, was collected from a fourth randomly chosen quadrat ( 4 sites $\times 6$ biomass samples). Hitchcock and Cronquist [22] and Pojar and MacKinnon [23] were used to identify plant species.

\subsection{Sample Preparation and Analysis}

Soil was dried at $45^{\circ} \mathrm{C}$ for one week, sieved (screen $1 \mathrm{~cm} \times 1 \mathrm{~cm}$ ) to remove rocks greater than $1 \mathrm{~cm}$ in diameter. From each sieved soil sample $(\mathrm{n}=72)$, approximately $60 \mathrm{~g}$ was taken from each sample to make a homogenized composite sample representing each transect, totaling 24 composite samples. From each composite sample, a $30 \mathrm{~g}$ sub-sample was ground into a fine powder using a SPEX Mixer Mill in the Geology Department at WWU, which uses a hardened stainless-steel mortar and two stainless steel ball bearings. The ground composite sub-samples were used to test for P and CEC at Spectrum Analytical (Washington Court House, $\mathrm{OH}$ ). Soil organic matter from the composited soil samples was determined using an optimized weight loss-on-ignition (WLOI) methodology specific to estuarine sediment [24]. Soil was dried overnight at $110^{\circ} \mathrm{C}$ to remove any adsorbed water. Approximately two to three grams of soil was added to a ceramic crucible and the combined weight of the sample and crucible was recorded. The samples were then placed in a muffle furnace at $550^{\circ} \mathrm{C}$ for four hours. Crucibles were removed and cooled for 10 minutes before being weighed. Carbon content was determined by percent WLOI and was calculated by determining the difference of final soil weight after samples were heated for four hours, from the initial soil weight.

\subsection{Carbon-to-Nitrogen Elemental Analysis}

Dried, homogenized soil and vegetation samples were analyzed for total carbon and nitrogen using a Thermo Electron NC Soil Analyzer Flash EA 1112 Series (Thermo Electron Corporation, Milan, Italy). A mass of approximately $100 \mathrm{mg}$ was placed in tin capsules and compressed to remove any air prior to carbonto-nitrogen analysis. A calibration curve for nitrogen was established using an analytical standard of atropine, which contained $48.4 \mathrm{~g} \cdot \mathrm{kg}^{-1}$ nitrogen. The calibration curve for nitrogen had an $\mathrm{R}^{2}$ value of 0.99 and the mass of atropine ranged from $0.05 \mathrm{mg}$ to $0.96 \mathrm{mg}$. The calibration curve for carbon also had an $\mathrm{R}^{2}$ value of 0.99 and the mass of atropine ranged from $0.77 \mathrm{mg}$ to $13.67 \mathrm{mg}$.

\subsection{Statistical Analyses}

A multiple analysis of variance (MANOVA) was used followed by a two-way fixed-effects analysis of variance (ANOVA) to compare percent carbon, soil organic matter, macro and micronutrients, species richness, species abundance using the Shannon-Wiener $\left(\mathrm{H}^{\prime}\right)$, and number of invasive species occurrences at 
each site. The fixed effects were site (pre, 3-Yr, 9-Yr, and 12-Yr) and location (above and below) with an interaction term. Levene's test and Shapiro-Wilk's was used to assess how well data fit the assumption of homogeneity of variances and normally distributed populations, respectively. A log transformation was applied, when needed, prior to the ANOVA. A post-hoc pairwise comparison was used for sites and locations with significant ANOVA results $(P$-value $<0.05)$ using the "holm" $P$-adjustment method. When data could not be transformed to meet the assumptions of ANOVA, a Kruskal-Wallis non-parametric test was used.

The Chao and bootstrap population estimations were used to assess sampling efficiency. Principal components analysis (PCA) was used to determine which combination of species explained the most variance in the plant communities [25]. Because several species comprised nearly 20 percent of the entire vegetation population across all sites, a row-centered and scaled PCA was used to create homoscedasticity in the dataset, and thus scale species with large counts. Using the prcomp function in $\mathrm{R}$, based on a singular value decomposition of the data matrix, identification of which components accounted for the most variation and separation in each principal component was determined. Analysis of similarity (ANOSIM) was used to calculate dissimilarity by using a ranked dissimilarity matrix. Based on these assumptions, a two-way ANOSIM was calculated between site and location, with 1000 permutations. All data was analyzed by $\mathrm{R}$ Version 3.3.0; [26].

\section{Results}

\subsection{Percent Soil Carbon, Organic Matter, and Nutrients}

Cation exchange capacity (CEC) differed per site with higher levels associated with the oldest restoration sites and the and pre-site and lowest levels recorded in sediments associated with the 3-year-old sites, below the bridge $(P=0.002)$. The newest restoration site (3-Yr) below the point of culvert restoration was significantly lower in soil carbon when compared across the other sites. Pair-wise post-hoc tests indicate similarities between the pre-restoration site and the oldest post-restoration site (12-Yr) with intermediate values observed from the 9-Yr plots. There were also significant differences in percent soil organic matter between sites $(P=0.003)$. Similar with soil carbon, the newest site (3-Yr) below the location of culvert restoration was significantly lower in soil organic matter when compared to the other sites (Table 1). There was a significant interaction between site and location (above and below) when concentrations of $\mathrm{N}$ and $\mathrm{P}$ were compared among the sites driven by a significant $\mathrm{N}$ and $\mathrm{P}$ nutrient decreases below the bridge at the 3 rd year, newest site $(P<0.05$; Table 1$)$.

\subsection{Vegetation}

No differences existed when plant height and aboveground biomass was compared among sites and locations. A significant difference between site was noted 
Table 1. Soil cation exchange capacity (CEC), percent soil carbon (\%C), soil organic matter (\%SOM), total percent nitrogen (\%N), phosphorus (P), and plant species richness and plant species diversity from four estuary restoration sites in Kitsap County, Washington. Samples were pooled for analysis from two distinct locations (above and below; $\mathrm{n}=23$ ) at each site: pre-restoration (pre), three (3-Yr), nine (9-Yr), and 12 years (12-Yr) post-restoration. Means connected by the same letter do not differ according to Tukey's HSD $(\alpha=0.05)$.

\begin{tabular}{|c|c|c|c|c|c|c|c|}
\hline & $\mathrm{CEC}(\mathrm{cmol} / \mathrm{kg})$ & $\% \mathrm{C}$ & $\%$ SOM & $\% \mathrm{~N}$ & $P(\mathrm{mg} / \mathrm{g})$ & Plant Sp. Diversity & Plant Sp. Richness \\
\hline \multicolumn{8}{|l|}{ Pre-site } \\
\hline Above & $12.5 \pm 0.4^{\mathrm{ab}}$ & $3.5 \pm 1.0^{\mathrm{a}}$ & $10.9 \pm 2.5^{\mathrm{ab}}$ & $0.2 \pm 0.1^{\mathrm{a}}$ & $60.7 \pm 2.3^{\mathrm{ab}}$ & $1.2 \pm 0.07^{\mathrm{a}}$ & $5 \pm 0.3^{\mathrm{a}}$ \\
\hline Below & $15.3 \pm 3.4^{\mathrm{a}}$ & $7.5 \pm 2.9^{\mathrm{a}}$ & $17.2 \pm 6.2^{\mathrm{ab}}$ & $0.5 \pm 0.2^{\mathrm{a}}$ & $127.0 \pm 28.6^{\mathrm{a}}$ & $1.5 \pm 0.2^{\mathrm{b}}$ & $7.3 \pm 1.5^{\mathrm{b}}$ \\
\hline \multicolumn{8}{|c|}{ 3-yr site } \\
\hline Above & $10.2 \pm 0.5^{\mathrm{ab}}$ & $2.4 \pm 0.4^{\mathrm{ab}}$ & $6.8 \pm 1.2^{\mathrm{ab}}$ & $0.2 \pm 0.1^{\mathrm{a}}$ & $222.3 \pm 65.3^{\mathrm{a}}$ & $1.1 \pm 0.1^{\mathrm{a}}$ & $5.5 \pm 0^{\mathrm{ab}}$ \\
\hline Below & $2.1 \pm 1.1 \mathrm{~b}$ & $0.9 \pm 0.5^{c}$ & $2.8 \pm 1.0^{c}$ & $0.03 \pm 0.01^{\mathrm{b}}$ & $35.7 \pm 8.7^{\mathrm{b}}$ & $1.4 \pm 0.07^{\mathrm{ab}}$ & $6.0 \pm 0.3^{\mathrm{ab}}$ \\
\hline \multicolumn{8}{|c|}{ 9-yr site } \\
\hline Above & $9.2 \pm 0.8^{\mathrm{ab}}$ & $2.0 \pm 0.3^{\mathrm{bc}}$ & $5.8 \pm 0.7^{\mathrm{ab}}$ & $0.1 \pm 0.01^{\mathrm{a}}$ & $36.7 \pm 3.5^{\mathrm{b}}$ & $1.6 \pm 0.04^{\mathrm{b}}$ & $10.7 \pm 0.8^{c}$ \\
\hline Below & $8.5 \pm 3.4^{\mathrm{ab}}$ & $2.2 \pm 0.7^{\mathrm{b}}$ & $6.8 \pm 2.2^{\mathrm{ab}}$ & $0.1 \pm 0.1^{\mathrm{a}}$ & $56.0 \pm 15.5^{\mathrm{ab}}$ & $1.5 \pm 0.01^{\mathrm{b}}$ & $7.7 \pm 0.4^{\mathrm{b}}$ \\
\hline \multicolumn{8}{|c|}{$12 y r$ site } \\
\hline Above & $16.6 \pm 2.3^{\mathrm{a}}$ & $2.6 \pm 0.9^{\mathrm{ab}}$ & $11.9 \pm 2.6^{\mathrm{ab}}$ & $0.2 \pm 0.1^{\mathrm{a}}$ & $92.7 \pm 6.9^{\mathrm{a}}$ & $2.0 \pm 0.06^{c}$ & $10.2 \pm 0.3^{c}$ \\
\hline Below & $14.4 \pm 3.0^{\mathrm{a}}$ & $4.6 \pm 1.1^{\mathrm{ab}}$ & $7.1 \pm 1.7^{\mathrm{ab}}$ & $0.3 \pm 0.1^{\mathrm{a}}$ & $129.7 \pm 52.8^{\mathrm{a}}$ & $2.0 \pm 0.07^{c}$ & $10.2 \pm 1.2^{\mathrm{c}}$ \\
\hline
\end{tabular}

Superscript letters indicate homogenous subsets, as determined by Tukey's HSD.

for plant species diversity by site $\left(\mathrm{F}_{(3,16)}=23.58, P<0.001\right.$; Table 1$)$. The prerestoration site was the only site with significant differences in plant species diversity between above vs. below location $(P<0.005)$. Additionally, the Shannon-Wiener $\left(\mathrm{H}^{\prime}\right)$ index of species diversity was significantly highest at the oldest post-restoration site $(1.98 \pm 0.04)$ than any other site. A trend toward a more diverse plant community assemblage can be seen over time. A similar trend was noted for plant species richness between site and location (Table 1).

Summer vegetation surveys documented a total of 64 plant species (Table 2). The Chao estimated $73( \pm 9)$ species followed by the bootstrap method, which estimated a population size of $66( \pm 3)$. The most abundant native plant species throughout all the sites included: pickleweed (Salicornia virginica; 19.2\%), orache (Atriplex patula; 9.5\%), gumweed (Grindelia squarrosa; 8.0\%), saltgrass (Distichlis spicata; 6.4\%), meadow grass (Hordeum brachyantherum; 4.9\%), and dune grass (Elymus mollis; 4.2\%). ANOSIM indicated significant dissimilarities between location (above and below) at all sites (All $P<0.05$; Table 3).

The principal components ordination was used to illustrate the vegetation community sampled during this study. Principal components I-II accounted for $32.3 \%$ of the total variance and illustrates salinity and vertical gradients based on species variable loadings $\left(\chi^{2}=23.4 ; \mathrm{df}=6 ; P<0.0001\right.$; Figure 2). Principal component I describes the species distribution by salt-tolerance, whereas salt tolerant species (illustrated by green ellipse) cluster in the upper left of the ordination and differentiate into freshwater marsh species (illustrated by blue ellipse) 
Table 2. Complete species list of 64 species recorded along transects $(n=24)$, including scientific name, common name, location (above and below), native status, and relative species abundance (\%) for all transect measurements located at four estuary restoration sites in Kitsap County, Washington including: one pre-restoration (Pre) site and three post restoration sites aged: three (3-yr), nine (9-Yr) and 12 years (12-Yr). Species are listed by their functional group and native status $(\mathrm{N}=\mathrm{Native}$ and naturalized; $\mathrm{NX}=$ Noxious).

\begin{tabular}{|c|c|c|c|c|c|c|}
\hline \multirow{2}{*}{ Species name } & \multirow{2}{*}{ Abbrev } & \multirow{2}{*}{ Common Name } & \multicolumn{2}{|c|}{ Site (Pre, 3, 9, and 12) } & \multirow{2}{*}{$\begin{array}{l}\text { Native } \\
\text { Status }\end{array}$} & \multirow{2}{*}{$\begin{array}{c}\text { Relative } \\
\text { Abundance (\%) }\end{array}$} \\
\hline & & & Above & Below & & \\
\hline Forbs and vines & & - & - & - & - & - \\
\hline Achillea millefolium & $A c M i$ & Yarrow & N/A & Pre & $\mathrm{N}$ & $<0.01$ \\
\hline Argentina egedii & $A r E g$ & Pacific Silverweed & $12-\mathrm{Yr}$ & $9-\mathrm{Yr}$ & $\mathrm{N}$ & 0.25 \\
\hline Cakile edentula & $C a E d$ & American Sea Rocket & $3-\mathrm{Yr}$ & $3-\mathrm{Yr}$ & $\mathrm{N}$ & 0.71 \\
\hline Atriplex patula & ChAl & Orache & All Sites & All Sites & $\mathrm{N}$ & 9.46 \\
\hline Cirsium arvense & CiAr & Canadian Thistle & $9-\mathrm{Yr}$ & N/A & NX & 0.13 \\
\hline Convolvulus arvensis & $\mathrm{CoAr}$ & Bindweed & N/A & $12-\mathrm{Yr}$ & NX & $<0.01$ \\
\hline Cuscuta pacifica & $\mathrm{CuPa}$ & Dodder & $3-\mathrm{Yr}$ & N/A & $\mathrm{N}$ & $<0.01$ \\
\hline Cytisus scoparius & $C y S c$ & Scotch Broom & $9-\mathrm{Yr}$ & $3-\mathrm{Yr}, 12-\mathrm{Yr}$ & NX & 0.46 \\
\hline Daucus carota & $\mathrm{DaCa}$ & Queen Anne's Lace & N/A & $12-\mathrm{Yr}$ & NX & $<0.01$ \\
\hline Equisetum hymale & $E q H y$ & Scouring Rush & $9-\mathrm{Yr}$ & N/A & $\mathrm{N}$ & 0.04 \\
\hline Galium aparine & $G a A p$ & Sticky Weed & N/A & $3-\mathrm{Yr}$ & $\mathrm{N}$ & $<0.01$ \\
\hline Grindelia squarrosa & $G r S q$ & Gumweed & All Sites & All Sites & $\mathrm{N}$ & 7.96 \\
\hline Honkenya peploides & $\mathrm{HoPe}$ & Seabeach Sandwort & N/A & $3-\mathrm{Yr}$ & $\mathrm{N}$ & 0.29 \\
\hline Hypochaeris radicata & HyRa & Hairy Cat's Ear & $3-, 9-, 12-\mathrm{Yr}$ & $9-\mathrm{Yr}, 12-\mathrm{Yr}$ & NX & 0.13 \\
\hline Jaumea carnosa & $\mathrm{JaCa}$ & Fleshy Jaumea & Pre & Pre, $12-\mathrm{Yr}$ & $\mathrm{N}$ & 1.63 \\
\hline Lathyrus odoratus & $\mathrm{LaOd}$ & Sweet Pea & N/A & Pre, $12-Y r$ & $\mathrm{~N}$ & 0.75 \\
\hline Leucanthemum vulgare & $\mathrm{LeVu}$ & Ox-Eye Daisy & N/A & $9-\mathrm{Yr}$ & NX & 0.08 \\
\hline Lotus corniculatus & LoCo & Bird's-foot Trefoil & N/A & $9-\mathrm{Yr}$ & $\mathrm{N}$ & 1.50 \\
\hline Montia linearis & $M o L i$ & Montia & Pre, $12-Y r$ & N/A & $\mathrm{N}$ & 0.33 \\
\hline Plantago lanceolata & PILa & English Plantain & $9-\mathrm{Yr}$ & $9-\mathrm{Yr}, 12-\mathrm{Yr}$ & $\mathrm{N}$ & 0.25 \\
\hline Plantago major & PIMa & Round Leaf Plantain & $9-\mathrm{Yr}$ & $9-\mathrm{Yr}$ & $\mathrm{N}$ & 0.08 \\
\hline Plantago maritima & PIMa.1 & Sea Plantain & $3-, 12-Y r$ & Pre, $12-Y r$ & $\mathrm{~N}$ & 1.83 \\
\hline Polygonum aviculare & PoAv & Knotgrass & $12-\mathrm{Yr}$ & $12-\mathrm{Yr}$ & $\mathrm{N}$ & 0.04 \\
\hline Polystichum munitum & $P o M u$ & Sword Fern & $9-\mathrm{Yr}$ & N/A & $\mathrm{N}$ & 0.04 \\
\hline Prunella vulgaris & $\operatorname{PrVu}$ & Self-Heal & $\mathrm{N} / \mathrm{A}$ & $9-\mathrm{Yr}$ & $\mathrm{N}$ & $<0.01$ \\
\hline Ranunculus repens & $R a R e$ & Creeping Buttercup & $9-\mathrm{Yr}$ & N/A & $\mathrm{N}$ & 0.04 \\
\hline Rumex acetosa & $R u A c$ & Sorrel & $\mathrm{N} / \mathrm{A}$ & $9-\mathrm{Yr}$ & $\mathrm{N}$ & 0.04 \\
\hline Rumex crispus & $\mathrm{RuCr}$ & Curly Dock & N/A & $9-\mathrm{Yr}$ & $\mathrm{N}$ & 0.04 \\
\hline Sagina maxima & SaMa & Coastal Pearlwort & $3-, 12-Y r$ & N/A & $\mathrm{N}$ & 1.54 \\
\hline Salicornia virginica & SaVi & Pickleweed & All Sites & All Sites & $\mathrm{N}$ & 19.21 \\
\hline Spergularia canadensis & $S p C a$ & Sand Spurry & $12-\mathrm{Yr}$ & Pre, 3-Yr & $\mathrm{N}$ & 2.38 \\
\hline Symphyotrichum subspicatum & SySu & Douglas Aster & $12-\mathrm{Yr}$ & N/A & $\mathrm{N}$ & 0.17 \\
\hline
\end{tabular}


Continued

\begin{tabular}{|c|c|c|c|c|c|c|}
\hline Tanacetum vulgare & $T a V u$ & Tansy & $9-\mathrm{Yr}$ & N/A & $\mathrm{NX}$ & $<0.01$ \\
\hline Trifolium wormskioldii & $\operatorname{Tr} W o$ & Red Clover & $9-\mathrm{Yr}$ & $12-Y r$ & $\mathrm{~N}$ & 0.38 \\
\hline Triglochin maritima & $\operatorname{TrMa}$ & Seaside Arrowgrass & $3-, 12-Y r$ & Pre, 3-Yr & $\mathrm{N}$ & 0.75 \\
\hline Graminoids & & - & & & - & - \\
\hline Agrostis capillaris & $\mathrm{AgCa}$ & Colonial Bentgrass & All Sites & All Sites & $\mathrm{N}$ & 11.79 \\
\hline Agrostis exarata & $A g E_{X}$ & Spike Bent Grass & $12-\mathrm{Yr}$ & N/A & $\mathrm{N}$ & 0.04 \\
\hline Ammophila arenaria & $A m A r$ & European Beachgrass & N/A & Pre & NX & $<0.01$ \\
\hline Calamagrostis canadensis & $\mathrm{CaCa}$ & Blue Joint Grass & $12-\mathrm{Yr}$ & N/A & $\mathrm{N}$ & 0.25 \\
\hline Deschampsia cespitosa & $D e C e$ & Tufted Hair Grass & Pre & N/A & $\mathrm{N}$ & $<0.01$ \\
\hline Distichlis spicata & $D i S p$ & Saltgrass & Pre, 3-, 12-Yr & Pre, $12-\mathrm{Yr}$ & $\mathrm{N}$ & 6.38 \\
\hline Elymus glaucus & $E l G l$ & Blue Wild Rye & $\mathrm{N} / \mathrm{A}$ & Pre, $12-\mathrm{Yr}$ & $\mathrm{N}$ & 0.29 \\
\hline Elymus mollis & ElMo & Dune grass & N/A & $3-\mathrm{Yr}$ & $\mathrm{N}$ & 4.17 \\
\hline Elymus repens & ElRe & Quack Grass & All Sites & All Sites & $\mathrm{N}$ & 1.25 \\
\hline Holcus lanatus & $\mathrm{HoLa}$ & Velvet Grass & $9-, 12-\mathrm{Yr}$ & $9-\mathrm{Yr}$ & $\mathrm{N}$ & 0.21 \\
\hline Hordeum brachyantherum & $\mathrm{HoBr}$ & Meadow Barley & $3-, 12-\mathrm{Yr}$ & Pre, 3-Yr & $\mathrm{N}$ & 4.92 \\
\hline Phalaris arundinacea & PhAr & Reed Canary Grass & $9-, 12-\mathrm{Yr}$ & $9-, 12-\mathrm{Yr}$ & $\mathrm{NX}$ & 0.46 \\
\hline Sedges and Rushes & & - & & & - & - \\
\hline Carex lyngbyei & CaLy & Lyngby Sedge & Pre, 3-, 12-Yr & N/A & $\mathrm{N}$ & 0.79 \\
\hline Eleocharis palustris & ElPa & Spike Rush & $9-\mathrm{Yr}$ & N/A & $\mathrm{N}$ & 0.13 \\
\hline Juncus effusus & JuEf & Common Rush & $9-\mathrm{Yr}$ & $9-, 12-\mathrm{Yr}$ & $\mathrm{N}$ & 0.33 \\
\hline Juncus gerardii & $J u G e$ & Saltmeadow Rush & Pre & Pre, $12-\mathrm{Yr}$ & $\mathrm{N}$ & 1.25 \\
\hline Woody plants & & - & & & - & - \\
\hline Acer macrophyllum & AcMa & Big Leaf Maple & $9-\mathrm{Yr}$ & Pre, 9-Yr & $\mathrm{N}$ & 0.58 \\
\hline Alnus rubra & $A l R u$ & Red Alder & $9-\mathrm{Yr}$ & $9-\mathrm{Yr}$ & $\mathrm{N}$ & 0.58 \\
\hline Oemleria cerasiformis & $\mathrm{OeCe}$ & Oso Berry & N/A & Pre & $\mathrm{N}$ & 0.67 \\
\hline Pinus contorta & PiCo & Shore Pine & N/A & $12-\mathrm{Yr}$ & $\mathrm{N}$ & 0.63 \\
\hline Pseudotsuga menziesii & $P s M e$ & Douglas Fir & N/A & Pre & $\mathrm{N}$ & 0.21 \\
\hline Robinia pseudoacacia & $R o P s$ & Black Locust & N/A & $9-\mathrm{Yr}$ & $\mathrm{N}$ & 0.42 \\
\hline Rosa nutkana & $R o N u$ & Nootka Rose & Pre & Pre, 9-Yr & $\mathrm{N}$ & 0.63 \\
\hline Rubus armeniacus & $R u A r$ & Himalayan Blackberry & Pre, 9-, 12-Yr & Pre, 9-, 12-Yr & $\mathrm{NX}$ & 1.25 \\
\hline Rubus ursinus & $R u U r$ & Trailing Blackberry & N/A & $9-\mathrm{Yr}$ & $\mathrm{N}$ & 0.42 \\
\hline Salix sitchensis & $\mathrm{SaSi}$ & Sitka Willow & $9-\mathrm{Yr}$ & N/A & $\mathrm{N}$ & 0.17 \\
\hline Salix hookeriana & $\mathrm{SaHo}$ & Hooker Willow & $\mathrm{N} / \mathrm{A}$ & $9-\mathrm{Yr}$ & $\mathrm{N}$ & 1.08 \\
\hline Symphoricarpos albus & SyAl & Snowberry & N/A & Pre, 9, 12-Yr & $\mathrm{N}$ & 0.08 \\
\hline Tsuga heterophylla & TsHe & Western Hemlock & N/A & $9-\mathrm{Yr}$ & $\mathrm{N}$ & 0.04 \\
\hline \multicolumn{7}{|l|}{ Other } \\
\hline Bare Ground & $B a G r$ & - & All Sites & All Sites & - & 9.00 \\
\hline Large Woody Debris & $L W D$ & - & All Sites & All Sites & - & 1.17 \\
\hline
\end{tabular}


Table 3. Analysis of similarity between locations (above and below) at each site: one pre-restoration (Pre) site and three post restoration sites aged: three (3-Yr), nine (9-Yr) and 12 years (12-Yr), within this study. The number of permutations equaled 999. High ANOSIM R-values indicate larger dissimilarity in vegetation composition between locations.

\begin{tabular}{|c|c|c|c|c|c|c|c|c|}
\hline ANOSIM & Pre & & $3-Y r$ & & 9-Yr & & $12-\mathrm{Yr}$ & \\
\hline R-Value & 0.43 & & 0.81 & & 0.56 & & 0.35 & \\
\hline$P$-Value & $0.007^{* *}$ & & $0.005^{* *}$ & & $0.002^{* *}$ & & $0.008^{* *}$ & \\
\hline \multirow[t]{5}{*}{ SIMPER } & Species & $\mathrm{P}$ & Species & $\mathrm{P}$ & Species & $\mathrm{P}$ & Species & $\mathrm{P}$ \\
\hline & Pickleweed & 0.01 & Pickleweed & 0.02 & Orache & 0.003 & Saltgrass & 0.003 \\
\hline & Fleshy Jaumea & 0.03 & Dune grass & 0.003 & Colonial Bentgrass & 0.009 & Coastal Pearlwort & 0.05 \\
\hline & Shore Pine & 0.03 & Gumweed & 0.00 & Gumweed & 0.01 & Gumweed & 0.02 \\
\hline & & & & & Common Rush & 0.02 & Pacific Silverweed & 0.04 \\
\hline
\end{tabular}

*Significant at $P<0.05 ;{ }^{*}$ Significant at $P<0.01$

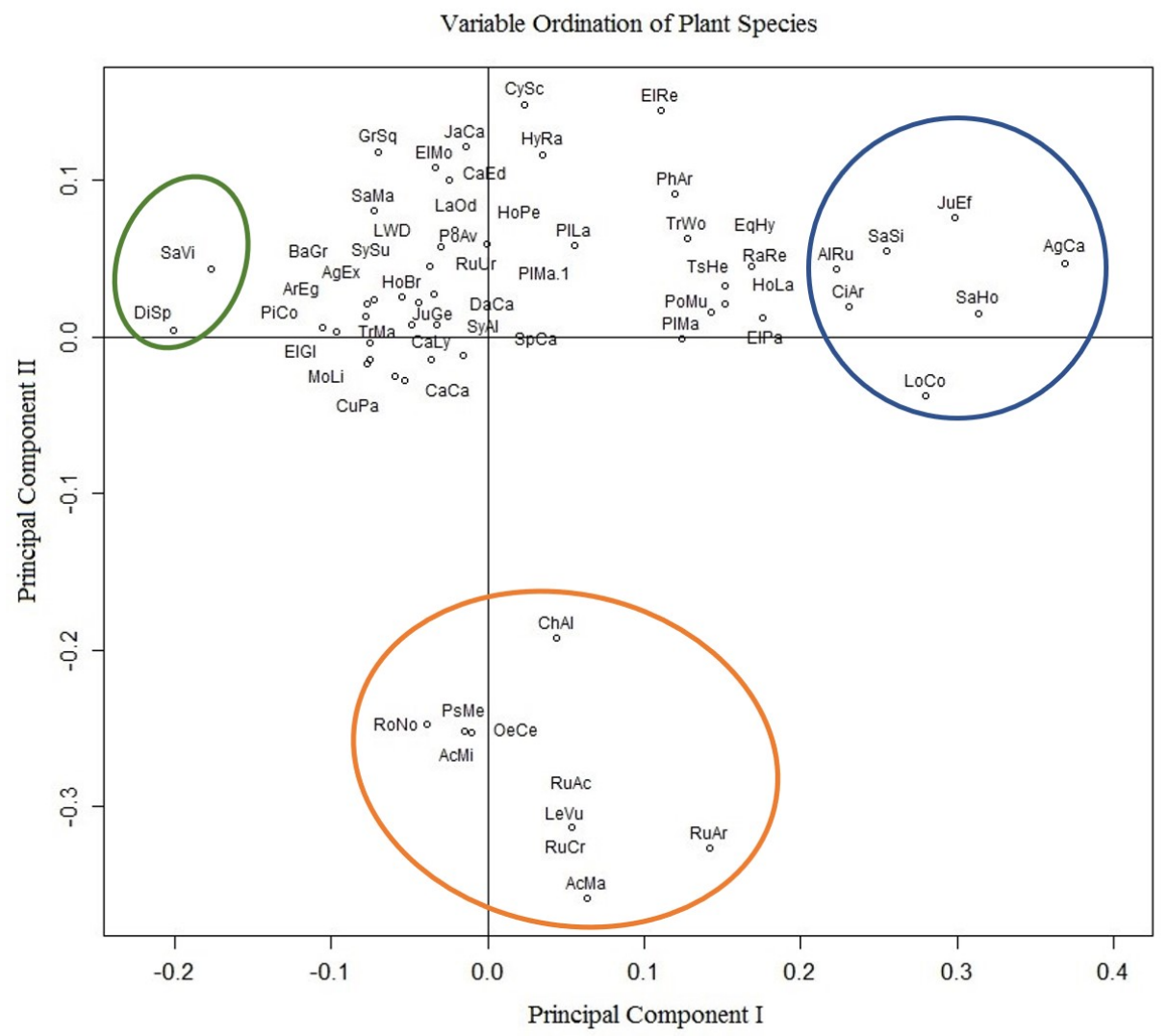

Figure 2. Variable ordination of principal component I and II (PCA) of plant species from four estuary restoration sites in Western Washington, USA. The four sites include one pre-restoration site (pre) and three post-restoration sites aged: three (3-Yr), nine (9-Yr), and 12 years (12-Yr). PCA by plant species using principal components I-IV from two distinct locations (above and below; $\mathrm{n}=24$ ). On PC I, the green ellipse indicated pickleweed ( $\mathrm{SaVi}$ ) and saltgrass (DiSp), two halophytic species. Those encircled by the blue ellipse, indicate species adapted to freshwater ecology. PC II is mainly separated by a vertical gradient, where species within the yellow ellipse form higher up on estuarine banks. All species abbreviations are defined in Table 2. 
into the right quadrate of the graph. Principal component II likely describes a vertical gradient, wherein older, woody native and non-native upland species (orange ellipse) differentiated from herbaceous marsh perennials and annuals (Figure 2).

Invasive species were relatively uncommon at each site and marginal evidence of a difference was observed in the interaction of site and location of the number of invasive occurrences in point-intercept data $\left(\mathrm{F}_{(3,40)}=2.52, P=0.07\right.$; Table 2). Nine invasive plant species were documented: Himalayan blackberry (Rubus armeniacus, 1.3\%), Scotchbroom (Cytisus scoparius, 0.5\%), reed canary grass (Phalaris arundinacea; 0.5\%), hairy cat's ear (Hypochaeris radicata; $0.1 \%$ ), Canada thistle (Cirsium arvense; $0.1 \%$ ), ox-eye daisy (Leucanthemum vulgare; $<0.1 \%$ ), European beachgrass (Ammophila arenaria; $<0.01 \%$ ), common tansy (Tanacetum vulgare; $<0.1 \%$ ), field bindweed (Convolvulus arvensis, $<0.1 \%$ ), and Queen Anne's lace (Daucus carota; $<0.1 \%)$.

\section{Discussion}

Differences in CEC, N, P, soil carbon and SOM existed among sites, with the lowest levels sampled from the newest post-restoration site. These results are consistent with other post-restoration sites [27], where large carbon deficits occur in initially restored systems [28] [29]. This reduction in soil carbon and organic matter could be due to several mechanisms. For one, heavy machinery and plant removal during bridge construction were responsible for displacing fine sediment and organic matter. Secondly, the reconnection of the estuary after culvert removal promoted movement by riverine outflows, daily tides, and weather events causing the displacement of lightweight, fine sediment particles into the estuary, leaving coarse sediment deposition at the tailwater of the new bridge. Estuaries are characterized by the dynamic deposition and resuspension of fine sediment particles; sediment accretion naturally occurs in the low-sloped areas when water velocity is reduced. Because the sites within this study are shallowwater estuaries with flat topography, velocity of silt and fine sediment slows and allows for the accretion of coarse sediment along estuarine edges near vegetation, which reduced the amount of fine sediment and SOM [30].

None of the sites displayed carbon or SOM concentrations representative of undisturbed, temperate coastal marshes on the eastern coasts of the U.S., which estimate normal soil carbon ranging between $12 \%-20 \%$ and organic matter ranging between $22 \%-35 \%$ [31]. Further, it is suggested that $18 \%-26 \%$ of estuarine soil (mineral or organic) is composed of organic matter [28], with $21 \%$ $25.6 \%$ of that organic matter being comprised of carbon [32]. Further, both $\mathrm{N}$ and $\mathrm{P}$ were deficient at the newest, lower restoration site. This is presumably linked to the lack of organic matter, which is required for adequate cation exchange capacity and nutrient/plant exchange [33]. This is especially true for nitrogen, which has been reported as deficient after 30 years post-restoration [29]. Authors note that climate and dominant plant community will differ for the west 
coast and influence soil carbon and organic matter differently; therefore, future studies that document Pacific Northwest projects over time will help better understand how restoration is improving ecosystem functioning.

Plant species diversity and richness increased over time, with the oldest postrestoration site (12-Yr) showing the highest species diversity of all study sites. Plants surveyed totaled 64 species between all sites and fell within the range of our statistical estimates. Forbs were the most common functional group sampled between all sites and comprised over half of species. Grasses, sedges, and rushes comprised just under one quarter of all species followed by woody species, which comprised $20 \%$ of all species sampled. Common estuarine species native to the $\mathrm{Pa}$ cific Northwest were encountered in relative proportion to functional group composition typical of a temperate pocket estuary, including non-native and non-tidal marsh plant species associated with culvert disturbance [34] [35].

Based on plants surveyed, pickleweed, saltgrass, orache, gumweed, and dunegrass are the most common at recent post-restoration sites, with gumweed, and dunegrass especially successful colonizing rocky, disturbed sites. The oldest post restoration site (12-Yr) had the highest species diversity and is relatively similar to other restored, temperate estuaries in terms of overall diversity based on Shannon-Weiner diversity index [36] [37]. Authors note that this site also had greater SOM, which may play roles in water relations and nutrient availability. This site harbored plants such as monita, sea plantain, coastal pearlwort, sand spurry, seaside arrowgrass and Pacific silverweed and may represent mid-successional species. Based on the community analyses, vegetation communities separated into two distinct groups: halophytic (salt tolerant) and glycophytic (salt intolerant), due to the formation of both a salinity and vertical gradient within the recovering floodplain and marshes. The salinity from the marine input is effective in deterring invasive species recruitment [38]. However, their presence was a result of a vertical gradient, which disconnected the plant community from the tidal flood plain, harbored non-native species. The second, glycophytic, group was composed of salt intolerant grasses, rushes, and early successional woody species. These were survey from areas of either strong freshwater influence, or also perched above the tidal flood plain. It is noted that species that appeared on the restoration planting lists were not sampled in this survey.

In summary, soil carbon and organic matter are initially lost during restoration with no sites having ranges comparable to natural estuaries. Borja et al. [27] suggest recovery from sediment modification and habitat creation is estimated to take at least two decades. Plant communities do not appear to homogenize between locations (above and below), due to the development of a salinity gradient, which is a common component of a natural estuary. However, vertical gradients at the shoreline resulted in perched vegetation communities that are removed from tidal influence, and conducive to woody riparian and non-native plant species establishment. Authors acknowledge these are results from an unreplicated, observational study that seeks to capture current state of local estuary 
soil and vegetation, which can be followed over time. This information hopes to inform future projects where direct seeding of pickleweed, saltgrass, seaside plantain, and Lyngby's sedge, can be initiated upon restoration. After the development of the salinity gradient, woody plantings (e.g. Nootka Rose, Oso Berry, Hooker's and Sitka Willow, Shore Pine) can be incorporated along the vertical gradient where inundation is infrequent. Time and additional sampling are required to determine the recovery of these estuaries over time, and if recovered floodplains to adequately create marsh habitat in lowland pocket estuaries required for the migration of anadromous fish species of Western Washington.

\section{Acknowledgements}

Authors thank Dr. Rebecca Bunn and Dr. Jim Helfield for written feedback when developing this manuscript. We also thank Dr. Peter Homann, Dr. Robin Matthews, Dr. Joan Hett, Christina Kereki and Christine Butler-Minor for their research support. Funding was provided by Western Washington University's Research and Sponsored Programs and Washington Department of Ecology.

\section{Conflicts of Interest}

The authors declare no conflicts of interest regarding the publication of this paper.

\section{References}

[1] Mitsch, W.J. and Gosselink, J.G. (2015) Wetlands. 5th Edition, John Wiley \& Sons, Inc., Hoboken.

[2] Beaumont, N.J., Austen, M.C., Atkins, J.P., Burdon, D., Degraer, S., Dentinho, T.P., Derous, S., Holm, P., Horton, T., van Ierland, E., Marboe, A.H., Starkey, D.J., Townsend, M. and Zarzycki, T. (2007) Identification, Definition and Quantification of Goods and Services Provided by Marine Biodiversity: Implications for the Ecosystem Approach. Marine Pollution Bulletin, 54, 253-266.

https://doi.org/10.1016/j.marpolbul.2006.12.003

[3] Craft, C.B., Broome, S.W. and Seneca, E.D. (1988) Nitrogen, Phosphorus and Organic Carbon Pools in Natural and Transplanted Marsh Soils. Estuaries, 11, 272280. https://doi.org/10.2307/1352014

[4] Marks, B.M., Chambers, L. and White, J.R. (2016) Effect of Fluctuating Salinity on Potential Denitrification in Coastal Wetland Soil and Sediments. Soil Science Society of America Journal, 80, 516-526. https://doi.org/10.2136/sssaj2015.07.0265

[5] Barbier, E.B. (2012) Progress and Challenges in Valuing Coastal and Marine Ecosystem Services. Review of Environmental Economics and Policy, 6, 1-19. https://doi.org/10.1093/reep/rer017

[6] Sheaves, M. (2009) Consequences of Ecological Connectivity: The Coastal Ecosystem Mosaic. Marine Ecology Progress Series, 391, 107-115. https://doi.org/10.3354/meps08121

[7] Craft, C. (2007) Freshwater Input Structures Soil Properties, Vertical Accretion, and Nutrient Accumulation of Georgia and U.S. Tidal Marshes. Limnology and Oceanography, 52, 1220-1230. https://doi.org/10.4319/lo.2007.52.3.1220 
[8] Fulford, R.S., Russell, M. and Rogers. J.E. (2016) Habitat Restoration from an Ecosystem Goods and Services Perspective: Application of a Spatially Explicit Individual-Based Model. Estuaries and Coasts, 39, 1801-1815. https://doi.org/10.1007/s12237-016-0100-6

[9] Huppert, D.D., Johnson, R.L., Leahy, J. and Bell, K. (2003) Interactions between Human Communities and Estuaries in the Pacific Northwest: Trends and Implications for Management. Estuaries, 26, 994-1009.

https://doi.org/10.1007/BF02803359

[10] Bartz, K.K., Lagueux, K.M., Scheuerell, M.D., Beechie, T., Haas, A.D. and Ruckelshaus, M.H. (2006) Translating Restoration Scenarios into Habitat Conditions: An Initial Step in Evaluating Recovery Strategies for Chinook Salmon (Oncorhynchus tshawytscha). Canadian Journal of Fisheries and Aquatic Sciences, 63, 1578-1595. https://doi.org/10.1139/f06-055

[11] D’Agostini, A., Gherardi, D.F.M. and Pezzi, L.P. (2015) Connectivity of Marine Protected Areas And Its Relation with Total Kinetic Energy. PLOS ONE, 10, e0139601. https://doi.org/10.1371/journal.pone.0139601

[12] Ward, J.V., Malard, F. and Tockner, K. (2002) Landscape Ecology: A Framework for Integrating Pattern and Process in River Corridors. Landscape Ecology, 17, 35-45. https://doi.org/10.1023/A:1015277626224

[13] Ellings, C.S., Davis, M.J., Grossman, E.E., Woo, I., Hodgson, S., Turner, K.L., Nakai, G., Takekawa, J.E. and Takekawa, J.Y. (2016) Changes in Habitat Availability for Outmigrating Juvenile Salmon (Oncorhynchus spp.) Following Estuary Restoration. Restoration Ecology, 24, 415-427. https://doi.org/10.1111/rec.12333

[14] Gray, A., Simenstad, C.A., Bottom, D.L. and Cornwell, T.J. (2002) Contrasting Functional Performance of Juvenile Salmon Habitat in Recovering Wetlands of the Salmon River Estuary, Oregon, U.S.A. Restoration Ecology, 10, 514-526. https://doi.org/10.1046/j.1526-100X.2002.01039.x

[15] Callaway, J. C. (2005) The Challenge of Restoring Functioning Salt Marsh Ecosystem. Journal of Coastal Research, 40, 24-36.

[16] Strange, E., Galbraith, H., Bickel, S., Mills, D., Beltman, D. and Lipton, J. (2002) Determining Ecological Equivalence in Service-to-Service Scaling of Salt Marsh Restoration. Environmental Management, 29, 290-300. https://doi.org/10.1007/s00267-001-0019-X

[17] Levine J.M., Vila, M., Antonio, C.M.D., Dukes, J.S., Grigulis, K. and Lavorel, S. (2003) Mechanisms Underlying the Impacts of Exotic Plant Invasions. Proceedings of the Royal Society B: Biological Sciences, 270, 775-781. https://doi.org/10.1098/rspb.2003.2327

[18] Clifton, B.C., Hood, W.G. and Hinton, S.R. (2018) Floristic Development in Three Oligohaline Tidal Wetlands after Dike Removal. Ecological Restoration, 36, 238-251. https://doi.org/10.3368/er.36.3.238

[19] Hobbs, R.J., Higgs, E. and Harris, J.A. (2009) Novel Ecosystems: Implications for Conservation and Restoration. Trends in Ecology and Evolution, 24, 599-605.

[20] USDA (2017) Web Soil Survey. Natural Resources Conservation Service. https://websoilsurvey.sc.egov.usda.gov/App/HomePage.htm

[21] Archer, E.K., Van Wagenen, E.R., Coles-Ritchie, M., Ojala, J.V., Roseen, T.P. and Gavin, A. (2016) Effectiveness Monitoring Sampling Methods for Riparian Vegetation Parameters. https://www.fs.usda.gov/Internet/FSE DOCUMENTS/fseprd494542.pdf 
[22] Hitchcock, C.L. and Cronquist, A. (1973) Flora of the Pacific Northwest: An Illustrated Manual. 5th Edition, University of Washington Press, Seattle.

[23] Pojar, J. and MacKinnon, A. (1994) Plants of the Pacific Northwest Coast: Washington, Oregon, British Columbia \& Alaska. Lone Pine Publishing, Vancouver.

[24] Wang, Q., Li, Y. and Wang, Y. (2001) Optimizing the Weight Loss-on-Ignition Methodology to Quantify Organic and Carbonate Carbon of Sediments from Diverse Sources. Environmental Monitoring and Assessment, 174, 241-257. https://doi.org/10.1007/s10661-010-1454-Z

[25] Gotelli, N.J. and Ellison, A.M. (2004) A Primer of Ecological Statistics. 2nd Edition, Sinauer Associates, Inc., Sunderland, MA.

[26] R Core Team (2016) R: A Language and Environment for Statistical Computing. R Foundation for Statistical Computing, Vienna. https://www.r-project.org/

[27] Borja, Á., Dauer, D.M., Elliott, M. and Simenstad, C.A. (2010) Medium and LongTerm Recovery of Estuarine and Coastal Ecosystems: Patterns, Rates and Restoration Effectiveness. Estuaries and Coasts, 33, 1249-1260. https://doi.org/10.1007/s12237-010-9347-5

[28] Craft, C., Broome S.W., Seneca E.D. and Showers, W.J. (1998) Estimating Sources of Soil Organic Matter in Natural and Transplanted Estuarine Marshes Using Stable Isotopes of Carbon and Nitrogen. Estuarine, Coastal and Shelf Science, 26, 633-641. https://doi.org/10.1016/0272-7714(88)90039-X

[29] Moreno-Mateos, D., Power, M.E., Comín, F.A. and Yockteng, R. (2012) Structural and Functional Loss in Restored Wetland Ecosystems. PLoS Biology, 10, e1001247. https://doi.org/10.1371/journal.pbio.1001247

[30] Fagherazzi, S., Carniello, L., D’Alpaos, L. and Defina, A. (2006) Critical Bifurcation of Shallow Microtidal Landforms in Tidal Flats and Salt Marshes. Proceedings of the National Academy of Sciences of the United States of America, 103, 8337-8341. https://doi.org/10.1073/pnas.0508379103

[31] Vincent, R.E., Burdick, D.M. and Dionne, M. (2013) Ditch-Plugging in New England Salt Marshes: Effects on Hydrology, Elevation, and Soil Characteristics. Estuaries and Coasts, 36, 610-625. https://doi.org/10.1007/s12237-012-9583-y

[32] Walsh, E.M., Ingalls, A.E. and Keil, R.G. (2008) Sources and Transport of Terrestrial Organic Matter in Vancouver Island Fjords and the Vancouver-Washington Margin: A Multiproxy Approach Using d13Corg, Lignin Phenols, and the Ether Lipid BIT Index. Limnology and Oceanography, 53, 1054-1063. https://doi.org/10.4319/lo.2008.53.3.1054

[33] Brady, N.C. (1974) The Nature and Properties of Soils. 8th Edition, Macmillan Publishing, Inc., New York.

[34] Seliskar, D. and Gallagher, J. (1983) The Ecology of Tidal Marshes of the Pacific Northwest Coast: A Community Profile. U.S. Fish and Wildlife Service, Division of Biological Services, Washington DC.

[35] Gabler, C.A., Osland, M.J., Grace, J.B., Stagg, C.L., Day, R.H., Hartley, S.B., Enwright, N.M., From, A.S., McCoy, M.L. and McLeod, J.L. (2017) Macroclimatic Change Expected to Transform Coastal Wetland Ecosystems This Century. Nature Climate Change, 7, 142-147. https://doi.org/10.1038/nclimate3203

[36] Craft, C., Reader, J., Sacco, J.N. and Broome, S.W. (1999) Twenty-Five Years of Ecosystem Development of Constructed Spartina Alterniflora (Loisel) Marshes. Ecological Applications, 9, 1405-1419. https://doi.org/10.1890/1051-0761(1999)009[1405:TFYOED]2.0.CO;2 
[37] Morgan, P.A. and Short, F.T. (2002) Using Functional Trajectories to Track Constructed Salt Marsh Development in the Great Bay Estuary, Maine/New Hampshire, U.S.A. Restoration Ecology, 10, 461-473.

https://doi.org/10.1046/j.1526-100X.2002.01037.x

[38] La Peyre, M., Greiner, K., Grace, J.B., Hahn, E. and Mendelssohn, I.A. (2001) The Importance of Competition in Regulating Plant Species Abundance along a Salinity Gradient. Ecology, 82, 62-69. https://doi.org/10.2307/2680086 